\title{
Time Evolution of the Microluminescence Energy of GaN/AlGaN Quantum Dots
}

\author{
K. SurowieckA*, A. Wysmołek, R. Stępniewski, R. Bożek, K. PakuŁa \\ AND J.M. BARANOWSKI \\ Institute of Experimental Physics, University of Warsaw, Hoża 69, 00-681 Warsaw, Poland
}

\begin{abstract}
Time evolution of the microphotoluminescence from low-density $\mathrm{GaN} / \mathrm{Al}_{x} \mathrm{Ga}_{1-x} \mathrm{~N}$ quantum dots grown by metal organic chemical vapor deposition using in situ etching of AlGaN is presented. The observed effect is related to the energy changes that begin immediately after sample illumination with the exciting laser light and saturate after some time. Typically, the luminescence energy decreases and the change is exponential with characteristic times in a range between several dozen and several hundred seconds. However, sometimes we observed the energy increase with characteristic times in a range between several and a few hundred seconds. The obtained results are discussed in terms of the metastable change of the electric field, induced by spontaneous polarization present in $\mathrm{GaN} / \mathrm{AlGaN}$ structure (in the growth direction), and strain- or defect-induced changes of the electric field in the vicinity of the dot.
\end{abstract}

PACS numbers: 78.55.Cr, 78.67.Hc

\section{Introduction}

Quantum dots are very promising candidates for a source of single photons, hence it is very important to understand processes that can influence their emission stability. Typically, the effects of system relaxation are studied in terms of time resolved photoluminescence (e.g. [1]). Our investigations were performed under continuous excitation. The phenomenon of a spontaneous change of quantum dots luminescence in such conditions is well known and was widely studied. Several groups reported energy jumps between discrete states, usually known as random telegraph noise $[2,3]$, as well as the spectral diffusion [4-7]. We have noticed both effects in the emission of $\mathrm{GaN} / \mathrm{AlGaN}$ quantum dots [8]. In this report, we discuss another type of the photoluminescence energy time evolution, recently found for this system. We have discovered that some of the observed emission lines exhibit monotonical energy changes, which begin immediately after illumination with the exciting laser and saturate after some time.

\section{Samples and experiment}

Low density $\mathrm{GaN} / \mathrm{Al}_{x} \mathrm{Ga}_{1-x} \mathrm{~N}(x \approx 0.1)$ quantum dots were grown by metal organic chemical vapor deposiotion (MOCVD) using a growth method based on in situ etching of the $\mathrm{AlGaN}$ surface in $\mathrm{SiH}_{4}$. The details of the growth procedure are described elsewhere [9]. Quantum

\footnotetext{
* corresponding author; e-mail: ksurow@fuw.edu.pl
}

dots obtained using this method can be observed with standard microphotoluminescence $(\mu \mathrm{PL})$ technique without any artificial mesas or mask structures.

Low temperature $(4.2-6.5 \mathrm{~K}) \mu \mathrm{PL}$ experiments were performed using continuous wave $\mathrm{He}-\mathrm{Cd}$ laser operating at $325 \mathrm{~nm}$ line that is slightly above the band gap of AlGaN layers in the investigated structures. The measurements were carried out using a continuous flow cryostat in the temperature range of $4.2-10 \mathrm{~K}$. The laser spot was focused on the samples using a reflecting microscope objective with the magnification of 25 . The size of the resulting excitation spot is estimated to be of about $3 \mu \mathrm{m}$ in diameter. The excitation power density was not higher than $10^{3}-10^{4} \mathrm{~W} / \mathrm{cm}^{2}$, thus the heating effects can be neglected.

\section{Results and discussion}

In this report, we focused on a group of quantum dots that emit light in the energies below the GaN band gap. In this spectral range, one can observe sharp, well resolved lines (see Fig. 1), which we assign to the exciton recombination in a dot. To study the effect of the emission energy change in detail, we measured a set of subsequent spectra in the same experimental conditions. A single spectrum was collected for a few (1-5) seconds and the measurement was continued up to a few hundred cycles.

For some lines, we noticed that luminescence energy is changing monotonically, typically it decreases, and that the observed evolution vanishes exponentially with characteristic times in a range between several dozen and several hundred seconds (see Fig. 1). However, sometimes 
we observed the energy increase with characteristic times in a range between several and a few hundred seconds. In the case of quantum dots ensemble, for different dots, negative and positive energy shifts with different characteristic times were observed simultaneously (data not shown). In some cases we can see the co-existence of the observed effects (Fig. 2), which suggests that their sources are independent and can be discussed in terms of metastable changes of the electric field of a different character.
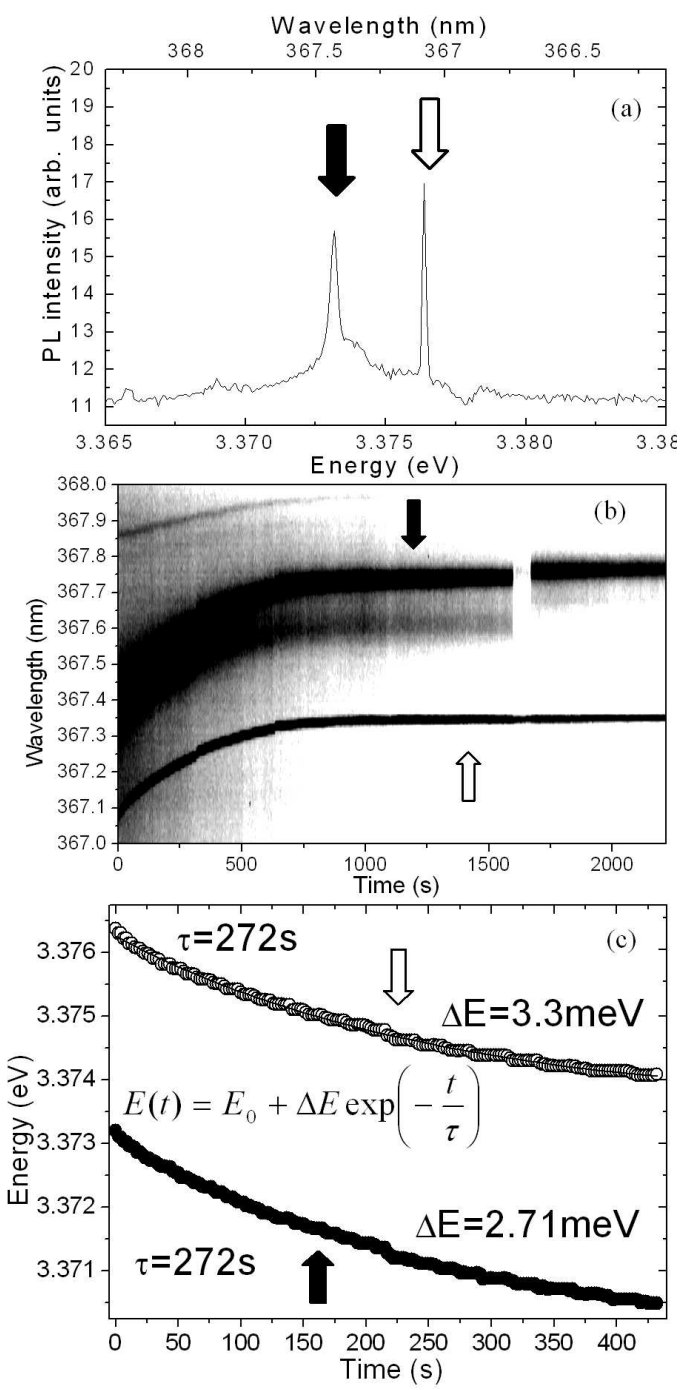

Fig. 1. The microphotoluminescence spectrum of investigated quantum dots (a). The map (b) shows the time evolution of the photoluminescence. $\mu \mathrm{PL}$ intensity is shown in a greyscale - from white (low) to black (high). Time dependence of the observed emission energy with the fitted formula and parameters is shown in $(c)$.

These changes are too small to modify significantly the transition probability of the observed lines. Indeed, for a single dot emission, we did not observe systematic correlations between the luminescence energy and the lumi-
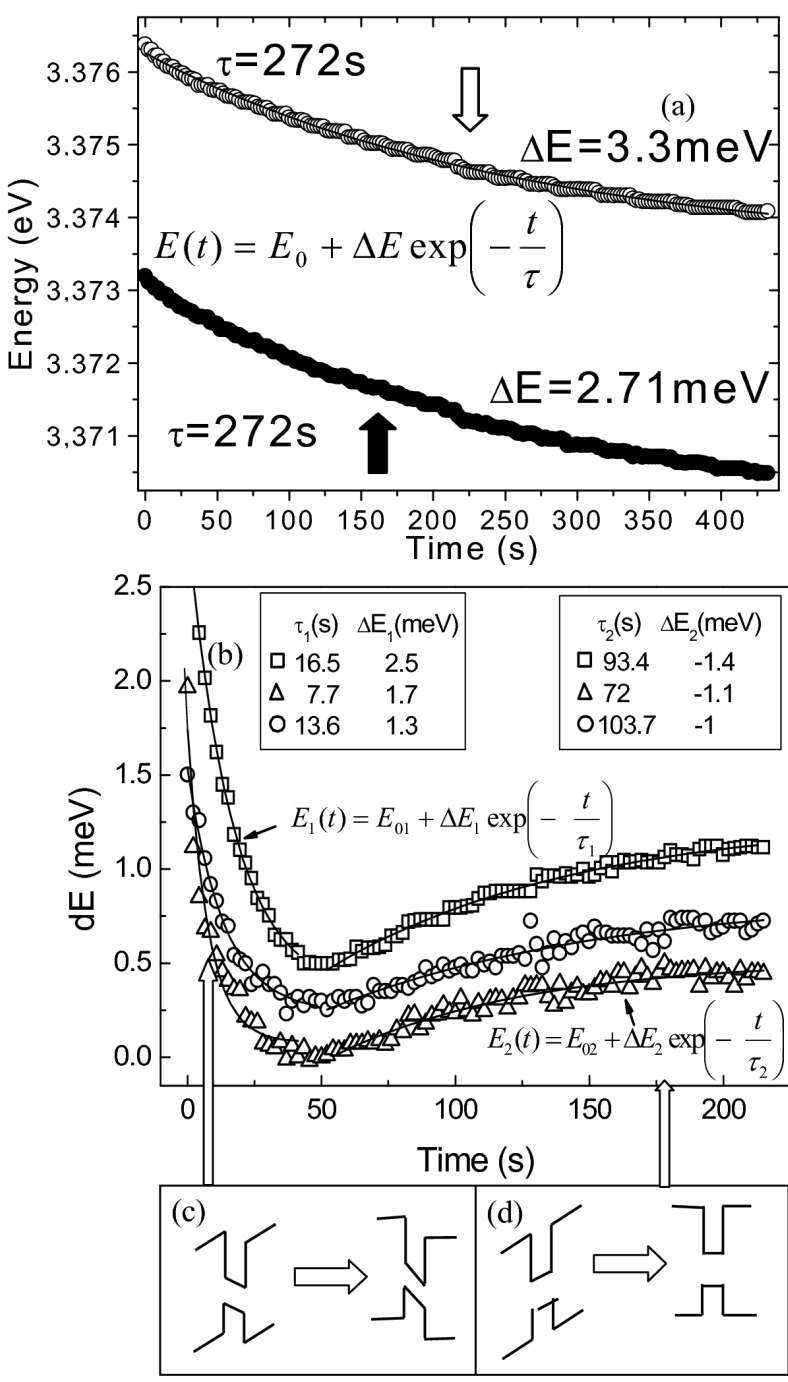

Fig. 2. The time evolution of the photoluminescence (a). $\mu \mathrm{PL}$ intensity is shown in a greyscale from white (low) to black (high). The changes of the energies for the traced $\mu \mathrm{PL}$ lines (arbitrarily shifted for clarity). (b) Two independent exponential decays were fitted and the fitting parameters are presented. (c) and (d) demonstrate the schemes of potential profile changes for the energy decrease and increase, respectively.

nescence intensity during the whole time of the evolution.

Typically, for a quantum well with a built-in electric field, the presence of photocreated carriers leads to (usually partial) electric field screening, and, consequently, to a blue shift of the luminescence energy. This effect is dynamic and can be observed either using a pulse excitation, or in experiments with different excitation power. In the first case, the initial blue shift disappears with time after the pulse excitation [1], which is attributed to the Stark shift recovery due to recombination of free carriers. In the second case, power dependence of the peak position (blue shift) is observed [10]. In our case, situation is quite different; the observed changes are rel- 
atively small in comparison to those reported in Ref. [1] and have a metastable character. If the illuminating laser was turned off and the sample was kept in dark for few minutes, the position of the observed lines remained unchanged. So, in the dark we notice a "freeeezing" of the sample state. Therefore, we connect observed effects with metastable changes of electric fields in the vicinity of the dot (barrier).

In $\mathrm{GaN} / \mathrm{AlGaN}$ structure, we deal with several sources of electric field. One of them is induced by piezo- and spontaneous polarization that is present in nitride structures. The other one has a random character and can be induced by a strain in a quantum dot or by capturing the carriers on the interface roughness or other defects.

Here, we have studied quantum dots luminescence in a spectral range below the GaN band gap. This results from the quantum confined Stark effect caused by the built-in electric field. Such a lowering of the emission energy shows that the observed objects are relatively big along the growth direction and can be treated similarly to the polar quantum wells. Electric field induced by piezo- and spontaneous polarization in GaN dots has opposite direction to the polarization present in the AlGaN barrier. Thus, the screening in the barrier induced by photocreated carriers leads to the increase of the electric field in the dot (well) and, as a consequence, the decrease of the emission energy is observed (see Fig. 2c).

The above situation is opposite to the one reported for non-polar quantum wells and dots [10], where the screening effect induced by photocreated carriers leads to the increase of the luminescence emission energy. Such a "classic" situation can be realised in our system when the electric field is perpendicular to the growth direction in the vicinity of a dot. In this case, the direction of the electric field in the quantum dot is the same as in the barrier (like in non-polar quantum wells), thus the screening process leads to the decrease of the electric field in the quantum dot (well) (see Fig. 2d). As a consequence, the increase of the luminescence energy is observed. Such a situation was reported previously for GaN/AlN quantum dots where, in consequence of the applied "in-plane" electric field, the decrease of the observed emission energy was seen [11].

\section{Conclusions}

We have shown that laser illumination of the structure can cause continuous and usually monotonical change of the energy in the investigated $\mathrm{GaN} / \mathrm{AlGaN}$ quantum dots. Typically, we observe exponential decrease of the energy, but sometimes exponential increase of the energy is seen. The observed change of the emission energy reaches a few $\mathrm{meV}$. It must be pointed OUT that in the range of the spot size we have observed different behaviour for different emission lines, thus the effect is very local.

\section{Acknowledgments}

This work was partially supported by research framework program MTKD-CT-2005-029671.

\section{References}

[1] T. Bretagnon, P. Lefebvre, P. Valvin, R. Bardoux, T. Guillet, T. Taliercio, B. Gil, N. Grandjean, F. Semond, B. Damilano, A. Dussaigne, J. Massies, Phys. Rev. B 73, 113304 (2006).

[2] M. Nirmal, B.O. Dabbousi, M.G. Bawendi, J.J. Macklin, J.K. Trautman, T.D. Harris, L.E. Brus, Nature (London) 383, 802 (1996).

[3] N. Panev, M.-E. Pistol, V. Zwiller. L. Samuelson, W. Jiang, B. Xu, Z. Wang, Phys. Rev. B 64, 045317 (2001).

[4] S.A. Empedocles, D.J. Norris, M.G. Bawendi, Phys. Rev. Lett. 77, 3873 (1996).

[5] F. Rol, S. Founta, H. Mariette, B. Daudin, Le Si Dang, J. Bleuse, D. Peyrade, J.-M. Gerard, B. Gayra, Phys. Rev. B 75, 125306 (2007).

[6] J. Rice, J. Robinson, A. Jarjour, R. Taylor, R. Olivier, G. Andrew, D. Briggs, M. Kappers, C. Humpreys, Appl. Phys. Lett. 84, 4110 (2004).

[7] J. Seufert, R. Weigand, G. Bacher, T. Kümmell, A. Forchel, K. Leonardi, D. Hommel, Appl. Phys. Lett. 76, 1872 (2000).

[8] K. Surowiecka, A. Wysmołek, R. Stępniewski, R. Bożek, K. Pakuła, J. Baranowski, Acta Phys. Pol. A 108, 879 (2005).

[9] K. Pakuła, R. Bożek, K. Surowiecka, R. Stępniewski, A. Wysmołek, J. Baranowski, J. Cryst. Growth 289, 472 (2006).

[10] W. Heller, U. Bockelmann, G. Abstreiter, Phys. Rev. B 57, 6270 (1997).

[11] T. Nakaoka, S. Kako, Y. Arakawa, Phys. Rev. B 73, 121305 (2005). 